\title{
A NOTE ON SPECIAL INVOLUTIONS
}

\author{
W. D. MUNN
}

(Received 19 December 1997; revised 7 June 1998)

Communicated by D. Easdown

\begin{abstract}
The algebra consisting of those linear transformations of a complex inner product space that have a formal adjoint is shown to possess a special involution. Two earlier results concerning special involutions are then generalized.
\end{abstract}

1991 Mathematics subject classification (Amer. Math. Soc.): 16S50, 16W10.

For a given complex inner product space $V$, the set of all linear transformations of $V$ that have a formal adjoint constitutes a complex algebra and is shown to possess an involution that is special in the sense of Easdown and Munn (Theorem 1). It follows that the standard involution on a $C^{*}$-algebra is special (Corollary 1 ) - a result first noted by Hofmann - and that hermitian conjugation is a special involution on the algebra of all $I \times I$ row-finite and column-finite complex matrices, where $I$ is an arbitrary nonempty set (Corollary 2). By combining Corollary 1 with a result of Barnes, it is proved that the natural involution on the $l^{1}$-algebra of an inverse semigroup is special (Theorem 2).

Let * be an involution on a semigroup $S$ (that is, a permutation of $S$ such that, for all $a, b$ in $S,(a b)^{*}=b^{*} a^{*}$ and $a^{* *}=a$ ). We say that ${ }^{*}$ is special if and only if, for each nonempty finite subset $T$ of $S$,

$$
(\exists t \in T)(\forall u, v \in T) \quad t^{*} t=u^{*} v \Rightarrow u=v .
$$

This definition is clearly equivalent to the one given originally in [2]. An involution [a special involution] on a complex algebra $R$ is a mapping ${ }^{*}: R \rightarrow R$ that is an automorphism of $(R,+)$ and an involution [a special involution] on $(R, \cdot)$, with the further property that, for all $a \in R$ and all $\lambda \in \mathbb{C}$ (the complex field), $(\lambda a)^{*}=\bar{\lambda} a^{*}$,

(C) 1998 Australian Mathematical Society 0263-6115/98 $\$ A 2.00+0.00$ 
where $\bar{\lambda}$ denotes the complex conjugate of $\lambda$. Examples of special involutions include hermitian conjugation on the algebra of all $n \times n$ complex matrices [2] and the natural involution on the complex semigroup algebra of an inverse semigroup [3]. Both of these are generalized here. By a star subalgebra of a complex algebra $R$ with an involution * we mean a subalgebra $S$ of $R$ such that $a^{*} \in S$ for all $a \in S$. Observe that if * is special and $S$ is a star subalgebra of $R$ then * induces a special involution on $S$.

In the theorem below we examine a certain subalgebra of the algebra of all linear transformations of a complex inner product space, namely the subalgebra consisting of all elements that possess a 'formal adjoint'.

THEOREM 1. Let $V$ be a complex vector space that admits an inner product $\langle\mid\rangle$, let $L(V)$ denote the algebra of all linear transformations of $V$ and let

$$
A(V):=\{a \in L(V):(\exists b \in L(V))(\forall x, y \in V)\langle a x \mid y\rangle=\langle x \mid b y\rangle\} .
$$

Then

(i) $A(V)$ is a subalgebra of $L(V)$,

(ii) to each $a \in A(V)$ there corresponds a unique $a^{*} \in A(V)$ such that, for all $x, y \in V,\langle a x \mid y\rangle=\left\langle x \mid a^{*} y\right\rangle$,

(iii) the mapping * $: A(V) \rightarrow A(V), a \mapsto a^{*}$ is a special involution.

PROOF. (i) This is routine.

(ii) Let $a \in A(V)$. Suppose that $b, c \in L(V)$ are such that, for all $x, y \in V$, $\langle a x \mid y\rangle=\langle x \mid b y\rangle=\langle x \mid c y\rangle$. Then, for all $y \in V,\langle(b-c) y \mid(b-c) y\rangle=0$ and so $(b-c) y=0$. Thus $b=c$. This establishes the existence of a unique $a^{*} \in L(V)$ such that, for all $x, y \in V,\langle a x \mid y\rangle=\left\langle x \mid a^{*} y\right\rangle$. Then, for all $x, y \in V$,

$$
\left\langle a^{*} x \mid y\right\rangle=\overline{\left\langle y \mid a^{*} x\right\rangle}=\overline{\langle a y \mid x\rangle}=\langle x \mid a y\rangle
$$

and so $a^{*} \in A(V)$. (This argument shows also that $a^{* *}=a$.)

(iii) It is easily checked that ${ }^{*}$ is an involution on $A(V)$ : we must prove that it is special.

Let $T$ be a nonempty finite subset of $A(V)$. Write

$$
U:=\{a-b: a, b \in T\} .
$$

We show first that there exists a linear functional $\chi$ on $A(V)$ such that

(1) $(\forall a \in A(V)) \quad \chi\left(a^{*} a\right)$ is real and nonnegative,

(2) $(\forall u \in U) \quad \chi\left(u^{*} u\right)=0$ implies $u=0$.

If $U=\{0\}$ we take $\chi$ to be the zero mapping. Suppose, therefore, that $U \neq\{0\}$. Let $u_{1}, u_{2}, \ldots, u_{n}$ be the nonzero elements of $U$. For each $r \in\{1,2, \ldots, n\}$, choose 
$x_{r} \in V$ such that $u_{r} x_{r} \neq 0$. We define $\chi: A(V) \rightarrow \mathbb{C}$ by

$$
(\forall a \in A(V)) \quad \chi(a):=\sum_{i=1}^{n}\left\langle a x_{i} \mid x_{i}\right\rangle .
$$

It is clear that $\chi$ is linear. To establish (1), we simply note that, for any $a \in A(V)$,

$$
\chi\left(a^{*} a\right)=\sum_{i=1}^{n}\left\langle a^{*} a x_{i} \mid x_{i}\right\rangle=\sum_{i=1}^{n}\left\langle a x_{i} \mid a x_{i}\right\rangle ;
$$

further, (2) holds, since, for $r \in\{1,2, \ldots, n\}$,

$$
\chi\left(u_{r}^{*} u_{r}\right)=\sum_{i=1}^{n}\left\langle u_{r} x_{i} \mid u_{r} x_{i}\right\rangle \geq\left\langle u_{r} x_{r} \mid u_{r} x_{r}\right\rangle>0 .
$$

Choose $t \in T$ such that $\chi\left(t^{*} t\right)=\max \left\{\chi\left(a^{*} a\right): a \in T\right\}$. Suppose that $t^{*} t=a^{*} b$, where $a, b \in T$. We complete the proof by showing that $a=b$. Since $t^{*} t=\left(a^{*} b\right)^{*}=$ $b^{*} a$, we have that $(a-b)^{*}(a-b)=a^{*} a+b^{*} b-2 t^{*} t$. Hence, by (1) and the choice of $t$,

$$
0 \leq \chi\left((a-b)^{*}(a-b)\right)=\chi\left(a^{*} a\right)+\chi\left(b^{*} b\right)-2 \chi\left(t^{*} t\right) \leq 0
$$

and so $\chi\left((a-b)^{*}(a-b)\right)=0$. But $a-b \in U$. Hence, by (2), $a=b$.

COROLlARY 1 (Hofmann). The standard involution on a $C^{*}$-algebra is special.

PROOF. It is sufficient to consider the case of the $C^{*}$-algebra $B(V)$ of all bounded linear operators on a complex Hilbert space $V$. Clearly $B(V)$ is a star subalgebra of $A(V)$ and the standard involution on $B(V)$ is the restriction of the involution * on $A(V)$. Since ${ }^{*}$ is special, the result follows.

Let $I$ be a nonempty set. An $I \times I$ complex matrix $\left[\alpha_{i j}\right]$ is said to be row-finite if and only if, for all $i \in I$, the set $\left\{j \in I: \alpha_{i j} \neq 0\right\}$ is finite (possibly empty). Similarly, $\left[\alpha_{i j}\right]$ is column-finite if and only if, for all $j \in I,\left\{i \in I: \alpha_{i j} \neq 0\right\}$ is finite. The set $\mathbb{C}_{I}$ of all $I \times I$ complex matrices that are both row-finite and column-finite is a complex algebra under the usual matrix operations and is closed under hermitian conjugation.

COROLLARY 2. Let I be a nonempty set. Then hermitian conjugation is a special involution on $\mathbb{C}_{\text {I }}$.

PROOF. Let $V$ denote the complex vector space consisting of all $I \times\{1\}$ 'column' vectors with at most finitely many nonzero entries. Then the mapping $\theta: \mathbb{C}_{I} \rightarrow L(V)$ defined by $\theta(a) x=a x(x \in V)$, where $a x$ is the usual matrix product, is an injective 
homomorphism. Moreover, $V$ admits an inner product $\langle\mid\rangle$ defined by $\langle x \mid y\rangle=\Sigma_{i} \xi_{i} \bar{\eta}_{i}$, where $\xi_{i}$ and $\eta_{i}$ denote the $i$ th components of $x$ and $y$ respectively; and it is easily seen that, for all $a \in \mathbb{C}_{l}$ and all $x, y \in V,\langle a x \mid y\rangle=\left\langle x \mid a^{\dagger} y\right\rangle$, where $a^{\dagger}$ denotes the hermitian conjugate of $a$. Thus, for all $a \in \mathbb{C}_{l}, \theta(a) \in A(V)$ and $(\theta(a))^{*}=\theta\left(a^{*}\right)$. But, by the theorem, ${ }^{*}$ is special. Hence, since $\operatorname{im} \theta$ is a star subalgebra of $A(V)$ and $\theta$ is injective, it follows that hermitian conjugation is a special involution on $\mathbb{C}_{1}$.

Observe that if $I$ is infinite then $\operatorname{im} \theta$ above contains unbounded linear operators on $V$.

Each of these corollaries generalizes the result, due to Lavers [2, Example 4], that, for any positive integer $n$, hermitian conjugation is a special involution on the algebra of all $n \times n$ complex matrices.

A further application of Theorem 1 arises in the context of certain Banach algebras. Let $S$ be a semigroup. We denote by $l^{\prime}(S)$ the Banach algebra consisting of all functions $a: S \rightarrow \mathbb{C}$ of countable support such that $\Sigma_{x \in S}|a(x)|<\infty$, where addition and scalar multiplication are the usual pointwise operations, multiplication is convolution, and the norm \|\| is defined by

$$
\left(\forall a \in l^{1}(S)\right) \quad\|a\|:=\sum_{x \in S}|a(x)| .
$$

Now suppose that $S$ is an inverse semigroup; thus, to each $x \in S$ there corresponds a unique element $x^{-1} \in S$ (the 'inverse' of $x$ ) such that $x x^{-1} x=x$ and $x^{-1} x x^{-1}=x^{-1}$. It is well known that inversion $\left(x \mapsto x^{-1}\right)$ is an involution on $S$. As is readily checked, inversion on $S$ induces an involution ${ }^{\dagger}$ on $l^{1}(S)$ by the rule that

$$
(\forall x \in S) \quad a^{\dagger}(x):=\overline{a\left(x^{-1}\right)} .
$$

We now combine Corollary 1 with a result of Barnes [1] to show that ${ }^{\dagger}$ is special.

THEOREM 2 (Crabb). Let $S$ be an inverse semigroup. Then the involution on $I^{\prime}(S)$ induced by inversion on $S$ is special.

ProOF. As above, let ${ }^{+}$denote the involution on $l^{1}(S)$ induced by inversion on $S$. By [1, Theorem 2.3], there exists a Hilbert space $V$ and a (continuous) injective homomorphism $\theta: l^{\prime}(S) \rightarrow B(V)$, the algebra of all bounded linear operators on $V$, such that, for all $a \in l^{1}(S),(\theta(a))^{*}=\theta\left(a^{*}\right)$, where ${ }^{*}$ denotes the standard involution on $B(V)$. But, by Corollary $1,{ }^{*}$ is special. Hence, $\operatorname{since} \operatorname{im} \theta$ is a star subalgebra of $B(V)$ and $\theta$ is injective, it follows that ${ }^{*}$ is special.

This extends [3, Theorem 5.1]. 


\section{Acknowledgments}

I am grateful to K. H. Hofmann and M. J. Crabb for supplying the results in Corollary 1 and Theorem 2 respectively.

\section{References}

[1] B. A. Barnes, 'Representations of the $l$ '-algebra of an inverse semigroup', Trans. Amer. Math. Soc. 218 (1976), 361-396.

[2] D. Easdown and W. D. Munn, 'On semigroups with involution', Bull. Austral. Math. Soc. 48 (1993), 93- 100 .

[3] D. Easdown and W. D. Munn, 'Trace functions on inverse semigroup algebras', Bull. Austral. Math. Soc. 52 (1995), 359-372.

Department of Mathematics

University of Glasgow

Glasgow G12 8QW

Scotland

U.K.

e-mail: wdm@maths.gla.ac.uk 